\title{
Supporting Information: Gas Transport in Interacting Planar Brushes
}

Sabin Adhikari, ${ }^{\dagger}$ Arash Nikoubashman,${ }^{\ddagger}$ Ludwik Leibler, ${ }^{\top}$ Michael Rubinstein, ${ }^{\S}$ Jiarul Midya, ${ }^{\ddagger}$ and Sanat K. Kumar*,

$\dagger$ Department of Chemical Engineering, Columbia University, New York, NY 10027, USA $\ddagger$ Institute of Physics, Johannes Gutenberg University Mainz, Staudingerweg 7, 55128 Mainz, Germany

๑UMR Gulliver 7083 CNRS, ESPCI Paris, PSL Research University, 10 rue Vauquelin, 75005 Paris, France

$\S$ Department of Mechanical Engineering and Materials Science, Biomedical Engineering, Chemistry, and Physics, Duke University, Durham, NC 27708-0300, USA

E-mail: sk2794@columbia.edu 


\section{Derivation of zero osmotic pressure condition}

Consider a polymer solution comprised of $n_{p}$ chains, each with $\mathcal{N}$ Kuhn-segments, and there are $n_{0}$ solvent molecules so that the total number of solvent molecules and Kuhn-segments together becomes $n=n_{p} \mathcal{N}+n_{0}$. If a solvent molecule as well as a Kuhn-segment occupy a volume $v$, then assuming incompressibility, the polymer volume fraction, $\phi$, is

$$
\phi=\frac{n_{p} \mathcal{N}}{n}=\frac{n_{p} \mathcal{N} v}{V},
$$

where $V$ is the total volume of the system. Then the polymer osmotic pressure, $P$, is given by

$$
P=-\left(\frac{\partial F}{\partial V}\right)_{n_{p}, T},
$$

where $F$ is the total free energy and $T$ is temperature. Now, if $f$ be the free energy per lattice, $F=f n$. In Eq. (2), substituting $F$ by $f n$, then replacing both $n$ and $V$ by using Eq. (1), and performing the partial derivative we get

$$
P=-\frac{1}{v}\left(f-\phi \frac{\partial f}{\partial \phi}\right) .
$$

Equating $P$ to zero gives

$$
f-\phi \frac{\partial f}{\partial \phi}=0 .
$$

Equilibrium monomer volume fractions for a melt and a planar brush are numerically calculated by using their free energy densities (from the main text) and Eq. (4), result is shown in Figure S1. 


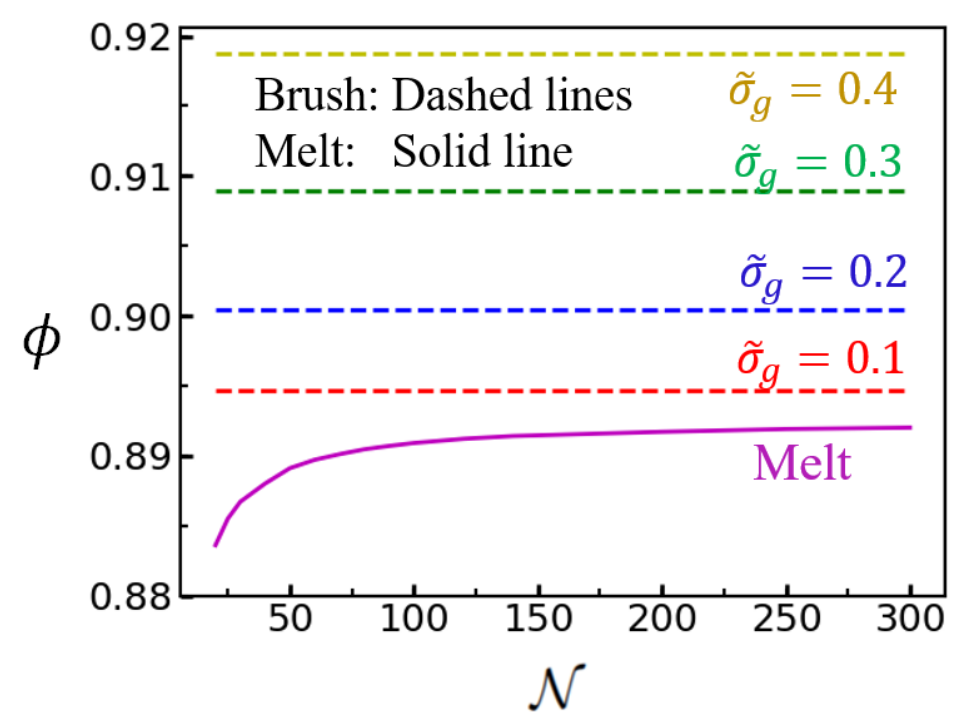

Figure S1: Monomer volume fraction $(\phi)$ versus chain length $(\mathcal{N})$ for a melt (magenta line), and a planar brush (dashed lines) at different grafting densities $\left(\tilde{\sigma_{g}}\right)$. It shows that the monomer density in a melt increases with increasing $\mathcal{N}$. However, the monomer density in a brush is independent of $\mathcal{N}$ while it increases with increasing $\tilde{\sigma_{g}}$.

\section{Local chain-elongation in interacting planar brushes}

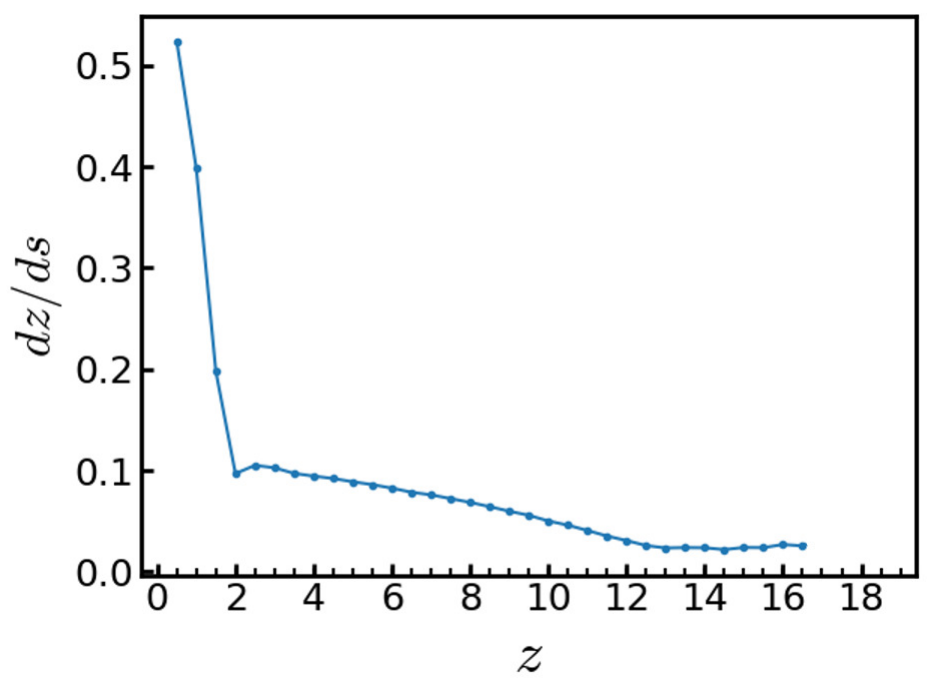

Figure S2: Local elongation of chains in the z-direction (perpendicular to the brush wall) as measured by the rate of net increment in $z$ with the chain contour length coordinate $s$. When the chain has no elongation, $\frac{d z}{d s}$ will be zero. Chain length $N=100$ and grafting density $\sigma_{g}=0.1 \sigma^{-2}$. The plot shows that for this set of $N$ and $\sigma_{g}$, chains are significantly extended only near the wall. 


\section{Chain-end probability distribution in interacting planar}

\section{brushes}

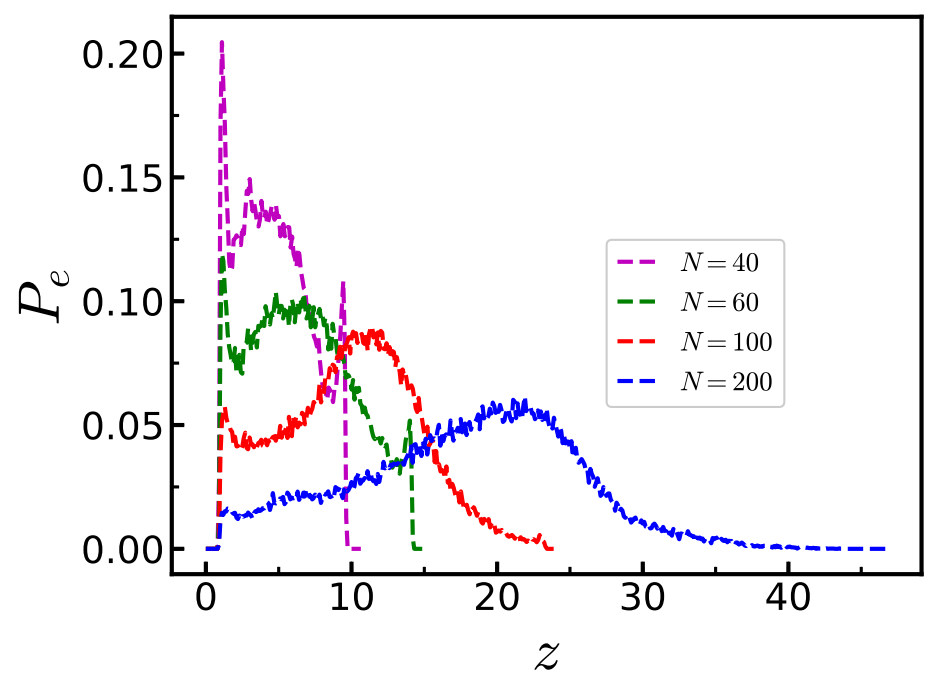

Figure S3: Chain-end probability distribution in interacting planar brushes for different chain lengths $N$ and a fixed grafting density $\sigma_{g}=0.1 \sigma^{-2}$. Note that chain-ends from only one brush are considered. Comparing these results with the corresponding results for a single brush (Figure 1 in the main text) shows that the chain-ends in a double brush are more broadly distributed, probably due to the mixing of chains from the opposite brushes in the interpenetration region.

\section{Tracers mean-square displacements (MSDs)}

Mean-square displacements (MSDs) for diffusion of tracer particles through a double-brush and a melt divided by time versus time is shown in Figure S4. 


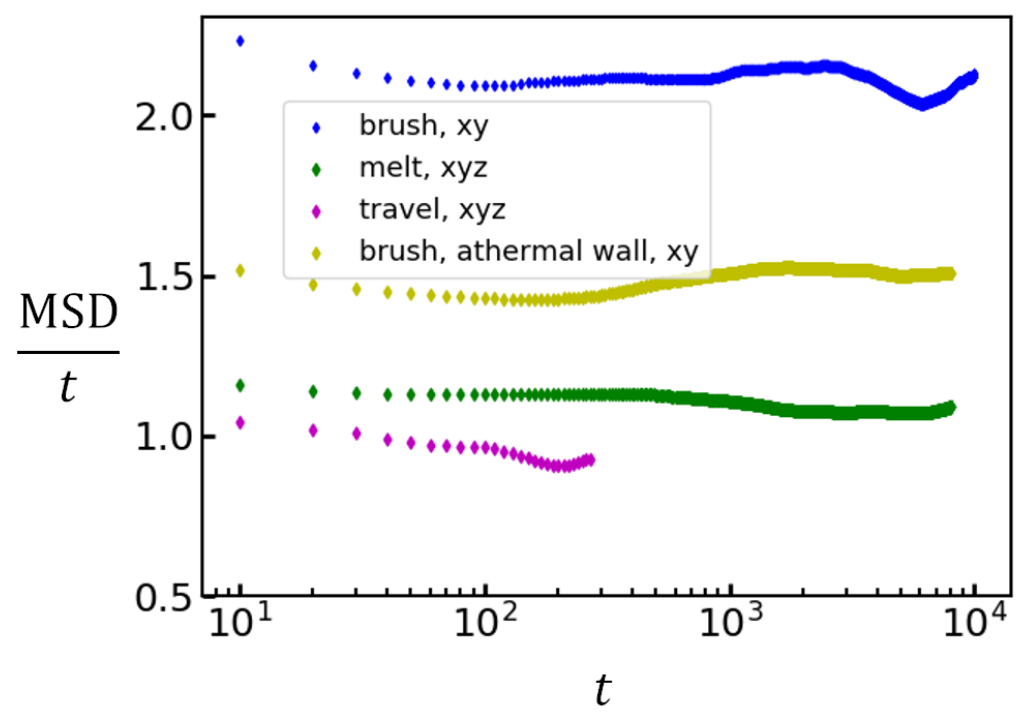

Figure S4: MSD/ $t$ versus $t$ ( $t$ is time) for diffusion of tracer particles in a double-brush and a melt. The blue symbols (brush, xy) show MSD on a plane parallel to the repulsive brush-walls while the magenta symbols (travel, xyz) show MSD during travels. The green symbols (melt, xyz) are for MSD in a melt and the yellow symbols (brush, athermal wall, xy) illustrate MSD on a plane parallel to the athermal brush-walls.

\section{Tracer particles 'travel' frequency}

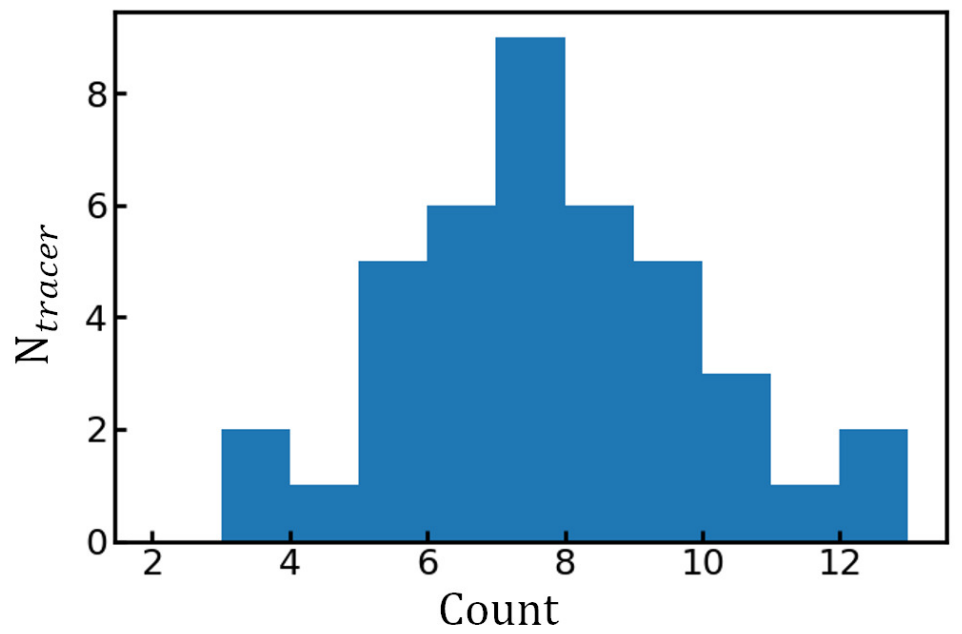

Figure S5: To test how long a tracer particle stays in the vicinity of a wall (defined by the threshold distance $2 \sigma$ ) once it reaches there, we followed the trajectories of 40 tracers for a time period of $20000 \tau$, and counted the number of travels from one brush to another that each tracer makes. The histogram shows that the majority of tracers travel from one brush to another around seven times (shown by 'Count' on the x-axis); no tracer stays only near a single wall for the entire time period. $N_{\text {tracer }}$ is the number of tracer particles with a given 'travel' frequency (or 'Count'). Once a tracer reaches near a wall, the time it takes to reach near the next wall is found to be $2740 \tau$, on average. 